\title{
Myoepithelial carcinoma with RB1 mutation: remarkable chemosensitivity to carcinoma of unknown origin therapy
}

Timothy M. Hoggard' ${ }^{1}$ Evita Henderson-Jackson 2,6, Marilyn M. Buii ${ }^{2,6}$, Jamie Caracciolo ${ }^{3}$, Jamie K. Teer ${ }^{4}$, Sean Yoder ${ }^{5}$, Odion Binitie ${ }^{6,8}$, Ricardo J. Gonzalez ${ }^{6}$ Andrew S. Brohl ${ }^{6}$ and Damon R. Reed ${ }^{6,7,8^{*}}$

\begin{abstract}
Background: Myoepithelial carcinoma of soft tissue is a rare, malignant neoplasm that is morphologically and immunophenotypically similar to its counterpart in salivary gland. It demonstrates myoepithelial differentiation, possessing both epithelial and myogenic characteristics. Thought to be chemotherapy insensitive, the optimal treatment regimen of this tumor has yet to be established and only a select few cases in the literature discuss treatment efficacy in detail.

Case presentation: Here we present a case of a young adult with metastatic myoepithelial carcinoma with an initial excellent response to systemic therapy utilizing carboplatin and paclitaxel with continued complete response after 3 years. The patient also underwent complete surgical excision and received adjuvant radiation to the primary site of disease. Exome sequencing revealed an inactivating mutation in RB1 which we believe to be the first such mutation to be reported in this cancer type.
\end{abstract}

Conclusions: Given increasing evidence suggesting RB1 loss is associated with responsiveness to conventional chemotherapies, particularly platinum-based regimens, we hypothesize that this genetic feature predisposed chemosensitivity in our patient's tumor.

Keywords: Myoepthelioid carcinoma, RB1, Chemotherapy, Paclitaxel, Carboplatin

\section{Background}

Myoepithelial tumors are rare salivary gland tumors classically found in the parotid gland. Most are benign myoepitheliomas. The malignant counterpart, myoepithelial carcinoma, is even more rare and represents less than $2 \%$ of salivary gland carcinomas [1]. Most cases of myoepithelial carcinoma are de novo in origin but may occasionally arise in association with a preexisting myoepithelioma or benign mixed tumor (pleomorphic adenoma) [2]. These malignant tumors also occur in non-salivary sites, such the nasopharynx, lung, breast, and skin [3-6]. About 50 cases of soft tissue locations of this tumor, both benign and malignant, have been described most often located in deep subcutaneous,

\footnotetext{
* Correspondence: damon.reed@moffitt.org

${ }^{6}$ Sarcoma Department, 12901 Bruce B Downs Blvd., Tampa, FL 33612, USA

${ }^{7}$ Chemical Biology and Molecular Medicine Program, 12901 Bruce B Downs

Blvd., Tampa, FL 33612, USA

Full list of author information is available at the end of the article
}

intramuscular, or subfascial tissue of the limbs and limb girdles [1, 6-10]. Compared to their salivary equivalent these tumors demonstrate increasing tendency for metastasis as well as aggressive histologic features, particularly within the pediatric population $[1,11]$. These tumors exhibit a heterogenous histomorphology and variable immunophenotypic findings, in turn, proving difficult to diagnosis [1]. Several recurrent molecular underpinnings unique to soft tissue myoepithelial carcinoma have been described, including EWSR1 gene rearrangements in up to $45 \%$ of cases [12]. Additionally, homozygous deletion of SMARCB1 has been reported in 3/5 cases that lack the EWSR1 gene rearrangement [13]. Comprehensive molecular analysis of this rare tumor type, however, has not been performed. 


\section{Materials and methods}

A chart review was conducted under IRB approval (MCC15003, University of South Florida IRB). To further evaluate our patient for a potential molecular explanation for dramatic chemotherapy response, we performed whole exome sequencing on the initial left popliteal mass resection, prior to any radiation or chemotherapy. Paired-end sequencing was performed on Illumina NextSeq $500(76 \times 2)$ instrument, generating $214,044,758$ total read pairs, resulting in $107 \times$ mean coverage across the capture region after duplication removal and mapping. 99.6\% of targeted bases achieved at least $10 \times$ depth of coverage. Burrows-Wheeler Aligner was used to align sequence reads to the human reference [14]. The Genome Analysis Toolkit was used for insertion/deletion realignment, quality score recalibration, and identification of single nucleotide and insertion/deletion variants [15]. To enrich for somatic mutations, we restricted our analysis to variants that are rare or absent in population databases (MAF $<0.01$ in 1000 Genomes Project, the NHLBI Exome Sequencing Project, and ExAC database). To further limit our findings to those most likely to be oncogenic, we utilized curated databases including COSMIC and the Cancer Gene Census to manually review variants for functional consequence and known status as an oncogene/tumor suppressor gene.

\section{Case presentation}

A 34-year-old male presented to our institution for evaluation of a left popliteal mass that was present and growing over 1 year with increasing pain. There was no neurologic or vascular compromise distal to the lesion. The patient developed inguinal pain 1 month prior to presentation. Otherwise the review of systems was negative.

Left knee MRI demonstrated a large, lobulated nonspecific T2-weighted hyperintense soft tissue mass in the popliteal fossa with local mass effect and surrounding soft tissue edema suspicious for soft tissue sarcoma (Fig. 1a). Contrast-enhanced computed tomography of the chest, abdomen, and pelvis performed for tumor staging demonstrated evidence of necrotic left external iliac lymphadenopathy (Fig. 1b), along with a right lung mass and a pulmonary nodule (Fig. 1c) most consistent with distant metastatic disease.

Tumor cells obtained from CT-guided core biopsy of the popliteal mass and then subsequently of the inguinal lymph nodes showed a proliferation of rounded epithelioid to spindle shaped cells with hyperchromatic nuclei arranged in trabecular-like architecture within hyalinized stroma. Ultimately, complete surgical resection of the primary, popliteal site was performed. Immunohistochemical evaluation revealed reactivity for vimentin, CAM5.2 as well as focal reactivity for CKAE1/3, EMA

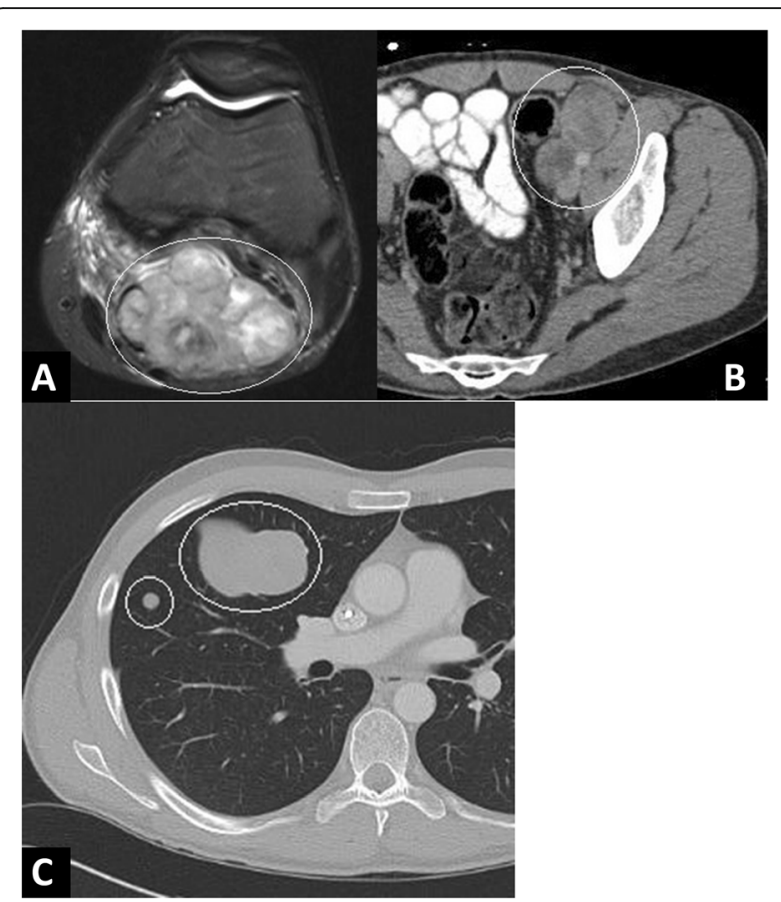

Fig. 1 Radiologic presentation. Upon initial presentation, (a) axial MRI (short tau inversion recovery/STIR) demonstrate a large lobulated soft tissue mass within the popliteal fossa, (b) axial contrast-enhanced CT images demonstrate bulky, necrotic left external iliac lymph nodes, and (c) axial CT images demonstrate a dominant right lung mass and small nodule consistent with pulmonary metastases

and synaptophysin. The tumor was negative for S-100, desmin, chromogranin and CD45. Further immunohistochemical analysis following external consultation revealed pankeratin and focal EMA positivity while staining for GFAP, calponin, p63, CD99, FLI-1, CD34, MUC-4, ERG and TLE-1 was negative. Given the limited sample, the lesion was tentatively termed "atypical spindle and round cell neoplasm, possibly myoepithelial in type" (Fig. 2a-f). The patient underwent radical resection of the popliteal mass with a positive margin allowing sufficient tissue to confirm the diagnosis. Grossly, the tumor measured $9.0 \times 7.8 \times 5.0 \mathrm{~cm}$ and cut sections showed an encapsulated, pale white, rubbery, lobulated mass. Histopathologic examination revealed a lobulated, multinodular, infiltrative malignant neoplasm composed of cellular nodules of epithelioid tumor cells with hyperchromatic nuclei showing frequent mitoses arranged in a trabecular fashion. Small proportions of the nodules were hypocellular with tumor cells exhibiting less nuclear atypia and more prominent myxoid stroma. Tumor necrosis was present. The specimen was again sent for consultation and the staining profile mirrored that of the biopsy specimens, aside from focal desmin positivity. Molecular analysis was notably negative for rearrangement of EWSR1 (22q12) locus and rearrangement of SS18 


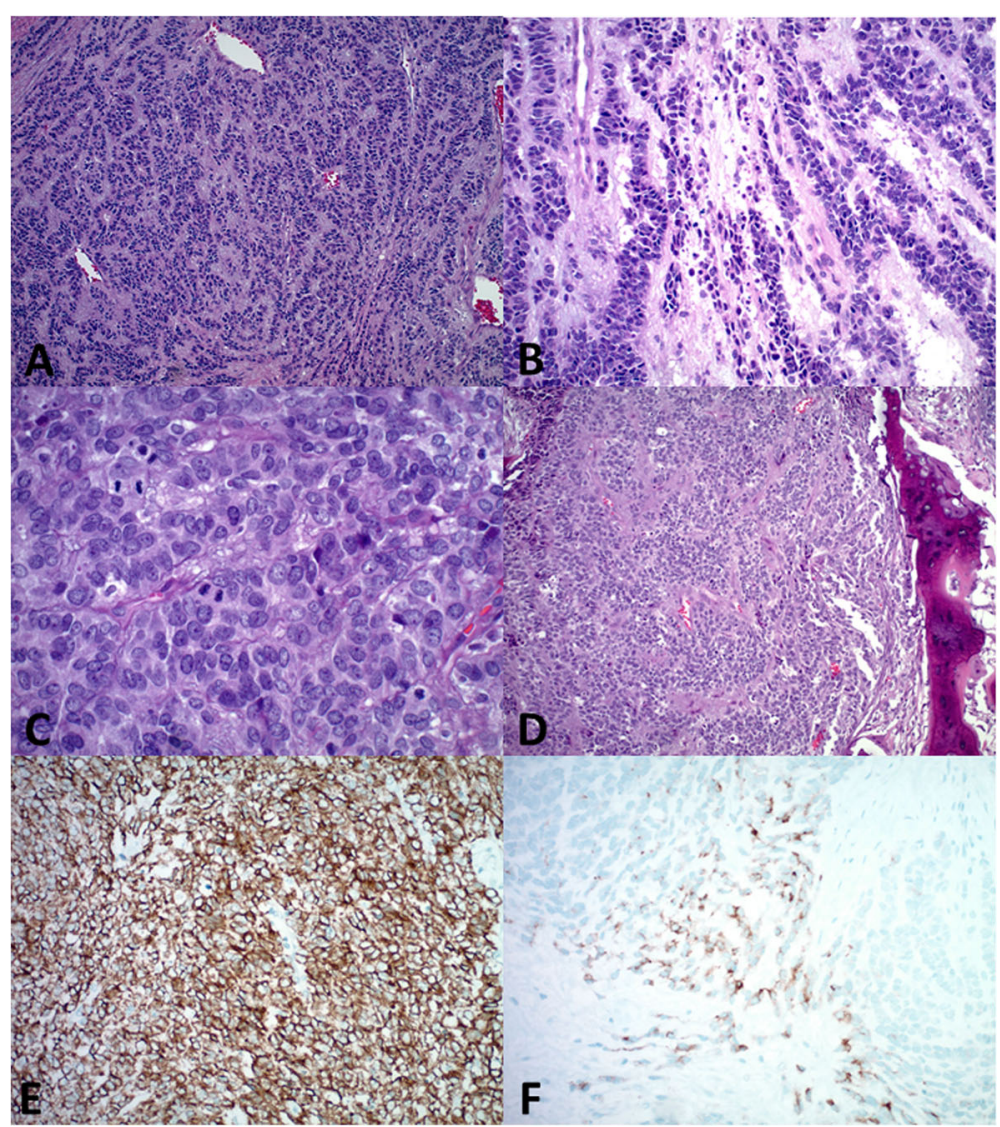

Fig. 2 Histologic analysis of tumor specimen at (a) 10x and (b) 20x magnification demonstrates rounded epithelioid to spindle shaped cells arranged in a trabecular-like fashion. (c) High power field demonstrates mitotic activity. (d) Bone formation is also noted. (e) Immunohistochemical analysis at 20x magnification reveals CKAE1/3 and CAM5.2 reactivity in addition to (f) focal EMA reactivity

(SYT; 18q11.2) locus. Additional molecular testing (FISH analysis) performed revealed no rearrangement of NR4A3. In view of the histomorphologic features and reactivity for epithelial markers, a final diagnosis of high-grade myoepithelial carcinoma was rendered both locally and by outside consultation, although the immunophenotype was not definitive in that regard.

Because of the systemic disease burden and limited reported activity of traditional sarcoma chemotherapeutic regimens, the case was discussed amongst medical and pediatric oncologists within and outside our institution without a clear consensus. We elected to treat with $3 \mathrm{cy}$ cles of carboplatin and paclitaxel initially with an almost immediate clinical response. Surveillance CT imaging of the chest, abdomen and pelvis demonstrated decreased size of iliac lymph nodes and pulmonary metastases consistent with tumor response to neoadjuvant therapy while MRI demonstrated surgical changes without clear, active disease (Fig. 3a-d).

The patient underwent a completion lymphadenectomy of the left superficial femoral and deep pelvic nodes without evidence of residual tumor in 25 examined lymph nodes. The patient received an additional 2 cycles of carboplatin and paclitaxel. Due to incomplete radiographic response the patient underwent a wedge resection, which also confirmed pathologic complete remission without malignancy identified. Hemorrhage and areas containing epithelioid macrophages with foamy and/or hemosiderin laden cytoplasm along focal adjacent hyaline fibrosis were seen. This was interpreted to be compatible with chemotherapy effect. The patient received adjuvant radiation therapy with 2 Gy fractions $\times 33$ doses to the popliteal fossa. The patient remains in radiographic remission 36 months from completion of chemotherapy. Molecular studies were undertaken to elucidate the mechanism responsible for the durable response to systemic therapy.

\section{Results}

From whole exome sequencing, we identified 509 highconfidence coding variants in our tumor specimen, including 45 truncating (missense, frameshift, or splice site) and 464 nonsynonymous. Of these, we identified 2 truncating mutations in well-described tumor suppressor genes, RB1 and MED12. We additionally note several 


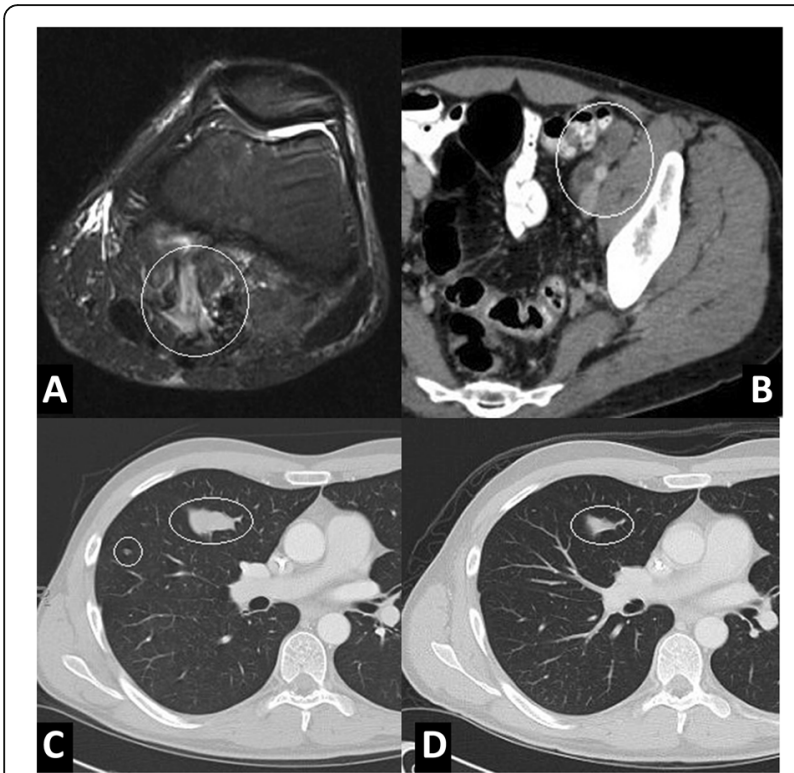

Fig. 3 Radiologic response to cisplatin and paclitaxel. a Axial MRI demonstrates postoperative changes following surgical resection of popliteal mass without evidence of residual disease. $\mathbf{b}$ Axial contrast-enhanced CT images following neoadjuvant chemotherapy demonstrate decreased size of left external iliac nodes, consistent with response to therapy. On chemotherapy at (c) 1 month and (d) 4 months after presentation, both the right lung mass and nodule have markedly improved consistent with response to therapy

mutations of less clear oncogenic consequence, including a truncating mutation in ITGA2, a possible tumor suppressor, and a nonsynonymous mutation in PDGFRA that has been reported in several cancer cases, but is also an uncommon population polymorphism [16-18]. Further description of these notable mutations is provided in Table 1.

\section{Discussion}

We report an exceptional and sustained response to chemotherapy in a young adult with myoepithelial carcinoma arising from the popliteal fossa with lymph node and pulmonary metastasis. While it is difficult to identify literature with a response rate for this rare malignancy or with a denominator of non-responders, there are at least case reports where chemotherapy regimens with broad activity have been attempted. Two such reports have shown either complete or partial response to

Table 1 Notable mutations in Myoepithelial Carcinoma tumor sample

\begin{tabular}{lll}
\hline Gene & Mutation & dbSNP \\
\hline RB1 & NM_000321:exon1:c.38_66del:p.A13fs & - \\
MED12 & NM_005120:exon13:c.C1861T:p.R621X & - \\
ITGA2 & NM_002203:exon17:c.G2155 T:p.E719X & - \\
PDGFRA & NM_006206:exon5:c.C661T:p.L221F & rs139913632 \\
\hline
\end{tabular}

carboplatin/paclitaxel $[5,6]$. Partial response in two adult patients is briefly mentioned in a myoepithelial tumor review from 2008, whereas a second report details a patient with a metastatic vulvar mass. Diagnosis in this case was made based upon immunohistochemical staining profile, which similarly was notable for CAM5.2 and focal CKAE1/3 reactivity. The patient was treated initially with excision of the mass and bilateral inguinal lymph node dissection followed by pelvic radiation, but a broad chemotherapy regimen utilizing carboplatin and paclitaxel was initiated after the development of pulmonary metastasis. A complete pathologic response was noted and the patient remained in complete remission over 3 years. An additional study showed complete response to both ifosfamide and melphalan in a patient with metastatic soft tissue disease [19]. In this report, the patient initially presented with a tumor in their toe, which subsequently recurred in the ipsilateral lower extremity following radical disarticulation. Hyperthermic isolated limb perfusion using tumor necrosis factor and melphalan was initiated with a complete response. Pelvic metastases were later noted, successfully treated with ifosfamide and radiation. Chemotherapeutic response in the pediatric population is similarly difficult to identify. In a series of 29 pediatric patients with soft tissue myoepithelial carcinoma, approximately half received chemotherapy [1]. Of these, only one patient demonstrated a clinical response, seen after multiple cycles of doxorubicin and ifosfamide due to metastasis following initial tumor excision. An additional series of 7 non-metastatic, pediatric patients reported favorable outcomes following a regimen utilizing cisplatin with six patients remaining without evidence of disease at a mean follow-up of 2.5 years [20]. Three of the seven tumors had EWSR1 rearrangement which was previously identified in series where it was associated with superficial location and more likely to be benign $[12,21]$. Our sequencing methodology would be unlikely to have detected an EWRS1 structural variation but clinical testing as mentioned above was negative.

Local surgical tumor excision with wide margins is recommended for myoepithelial carcinoma of soft tissues, although the optimal approach to treatment has yet to be established [6]. The efficacy of radiation or chemotherapy, either as an adjuvant therapy or in metastatic disease, also has not been consistently demonstrated $[1,6,11]$. As is common with rare malignancies, there is a lack of consensus guidelines and multiple options for care. In this case chemotherapy was incorporated because of the patients young age and metastatic presentation, but there are likely many scenarios whereby chemotherapy may be of benefit for patients with a similar malignancy whereby chemotherapy may not be considered. In fact, myoepithelial carcinoma is currently incorporated into a cooperative group clinical 
trial for soft tissue sarcomas as a chemotherapy resistant tumor eligible only for the "non-chemotherapy cohort" (NCT02180867).

Histologically, myoepithelial tumors often display a variety of cellular morphologies, making identification and diagnosis more difficult. The tumors may be composed exclusively of a single cell type, but are more frequently present as a combination of epithelioid, spindle cell, plasmacytoid or clear cell types [9]. Immunohistochemical staining serves as a key step in differentiating from similar appearing tumors. Myoepithelial carcinomas are generally positive for S-100, cytokeratin, epithelial membrane antigen (EMA) and $\alpha$-smooth muscle actin [9]. The differential diagnosis include carcinoma, melanoma, epithelioid sarcoma, extraskeletal myxoid chondrosarcoma and chordoma. EWSR1 gene rearrangement is identified in only $50 \%$ of the soft tissue myoepithlelial carcinoma [22].

Using whole exome sequencing, we examined our patient's tumor for possible oncogenic variants that may help elucidate a mechanism for chemotherapy sensitivity. Given that matched germline DNA was not available for comparison, we expect many of variants uncovered to be rare or private germline mutations or passenger somatic mutations and therefore of little oncogenic consequence. We were able to identify, however, truncating mutations in $R B 1$ and MED12 that are very likely to be somatic oncogenic drivers in this patient given the wellestablished role of these two genes as tumor suppressors across multiple tumor types. To our knowledge, this is the first description of inactivating mutation in either of these two genes reported in this cancer type.

The retinoblastoma protein $(R B 1)$ is one of the most frequently affected tumor suppressors across multiple cancer histologies and plays a critical role in regulation of cell cycle and apoptosis [23]. RB1 pathway deregulation has been reported in various benign and malignant salivary tumors, including malignant myoepithelioma [24]. Interestingly, preclinical and clinical evidence in multiple cancer types suggest that RB1 expressional loss is associated with increased responsiveness to conventional chemotherapies [23]. Additionally, a recent genomic study in small cell lung cancer showed that presence of $R B 1$ inactivating mutation was highly predictive of good response to platinum-based chemotherapy [25]. Childhood retinoblastoma, almost invariably caused by either germline or somatic mutational inactivation of $R B 1$, is also highly responsive to platinumbased chemotherapy [26]. Given this mounting evidence, we hypothesize that $R B 1$ mutation in our patient's tumor predisposed to chemosensitivity. In contrast, loss of the RNA polymerase II mediator complex member MED12 has been shown to induce drug resistance, particularly to tyrosine kinase inhibitor therapy, via activation of transforming growth factor B receptor signaling [27].
In summary, we report a case of myoepithelial carcinoma with a $R B 1$ inactivating mutation that experienced a dramatic response to platinum-based chemotherapy. We believe that our case adds to growing evidence across multiple cancer types that RB1 loss is predictive of chemosensitivity, perhaps in particular to platinumbased regimens. Given the rarity of this tumor type, the optimal systemic therapy approach is not well defined. Further study should be undertaken to evaluate whether RB1 loss is a recurring feature in this histology and whether platinum-based chemotherapy is more broadly effective in this tumor type outside of this case.

\section{Conclusion}

While formal recommendations are difficult to make based on a case report, our review of the literature would suggest that continued consideration for systemic carcinoma therapy, more specifically with paclitaxel and carboplatin, should be considered in myoepithelial carcinoma patients presenting with stage 4 disease and extremity primary locations.

\section{Abbreviations \\ COSMIC: Catalogue of Somatic Mutations in Cancer; CT: Computed Tomography; ExAC: Exome Aggregation Consoritum; IRB: Institutional Review Board; MAF: Minor allele frequency; MRI: Magnetic Resonance Imaging; NCT: National Clinical Trial; NHLBI: National Heart, Lung, and Blood Institute}

\section{Acknowledgements}

Our study received assistance from the Tissue Core, Molecular Genomics Core, and Cancer Informatics Core Facilities at the H. Lee Moffitt Cancer Center \& Research Institute, an $\mathrm{NCl}$ designated Comprehensive Cancer Center, supported under NIH grant P30-CA76292.

\section{Funding}

The study was funded by the Gonzmart Family Foundation. The funding body played no role in the design, conduct, interpretation, or writing of this research and manuscript.

\section{Availability of data and materials}

The datasets during and/or analyzed during the current study available from the corresponding author on reasonable request.

\section{Authors' contributions}

Substantial Contributions to conception and design, acquisition of data, analysis and interpretation of data: $\mathrm{TMH}, \mathrm{EH}, \mathrm{MMB}, \mathrm{JC}, \mathrm{SY}, \mathrm{OB}, \mathrm{RJG}, \mathrm{JKT}, \mathrm{ASB}$ and DRR. Been involved in drafting manuscript or revising it critically: TMH, $E H, M M B, J C, J K T, A S B$ and DRR. Given final approval for version to be published: TMH, EH, MMB, JC, SY, OB, RJG, JKT, ASB and DRR. Agree to be accountable: TMH, EH, MMB, JC, SY, OB, RJG, JKT, ASB and DRR. All authors read and approved the final manuscript.

\section{Competing interests}

The authors declare that they have no competing interests.

\section{Ethics approval and consent to participate}

The study was approved by the IRB of the University of South Florida, protocol MCC15003. We have obtained consent to publish from the individual in this case report.

\section{Publisher's Note}

Springer Nature remains neutral with regard to jurisdictional claims in published maps and institutional affiliations. 


\section{Author details}

'University of South Florida Morsani College of Medicine, 12901 Bruce B Downs Blvd., Tampa, FL 33612, USA. ²Department of Anatomic Pathology, 12901 Bruce B Downs Blvd., Tampa, FL 33612, USA. ${ }^{3}$ Department of Diagnostic Imaging, 12901 Bruce B Downs Blvd., Tampa, FL 33612, USA. ${ }^{4}$ Department of Biostatistics and Bioinformatics, 12901 Bruce B Downs Blvd., Tampa, FL 33612, USA. ${ }^{5}$ Molecular Genomics Core Facility, 12901 Bruce B Downs Blvd., Tampa, FL 33612, USA. ${ }^{6}$ Sarcoma Department, 12901 Bruce B Downs Blvd., Tampa, FL 33612, USA. ${ }^{7}$ Chemical Biology and Molecular Medicine Program, 12901 Bruce B Downs Blvd., Tampa, FL 33612, USA. ${ }^{8}$ Adolescent and Young Adult Program; H. Lee Moffitt Cancer Center and Research Institute, 12901 Bruce B Downs Blvd., Tampa, FL 33612, USA

Received: 13 October 2016 Accepted: 31 March 2017

Published online: 08 April 2017

\section{References}

1. Gleason BC, Fletcher CD. Myoepithelial carcinoma of soft tissue in children: an aggressive neoplasm analyzed in a series of 29 cases. Am J Surg Pathol. 2007:31:1813-24.

2. McCluggage WG, Primrose WJ, Toner PG. Myoepithelial carcinoma (malignant myoepithelioma) of the parotid gland arising in a pleomorphic adenoma. J Clin Pathol. 1998;51:552-6.

3. Miura K, Harada H, Aiba S, Tsutsui Y. Myoepithelial carcinoma of the lung arising from bronchial submucosa. Am J Surg Pathol. 2000;24:1300-4.

4. Dhawan A, Shenoy A, Sriprakash D. Myoepithelial carcinoma of the nasopharynx: case report of a rare entity. Natl J Maxillofac Surg. 2011;2:207-9.

5. Noronha V, Cooper DL, Higgins SA, Murren JR, Kluger HM. Metastatic myoepithelial carcinoma of the vulva treated with carboplatin and paclitaxel. Lancet Oncol. 2006;7:270-1.

6. Gleason BC, Hornick JL. Myoepithelial tumours of skin and soft tissue: an update. Diagnostic Histopathology. 2008;14:552-62.

7. Michal M, Miettinen M. Myoepitheliomas of the skin and soft tissues. Report of 12 cases. Virchows Arch. 1999:434:393-400.

8. Kilpatrick SE, Hitchcock MG, Kraus MD, Calonje E, Fletcher CD. Mixed tumors and myoepitheliomas of soft tissue: a clinicopathologic study of 19 cases with a unifying concept. Am J Surg Pathol. 1997:21:13-22.

9. Jo VY, Fletcher CD. Myoepithelial neoplasms of soft tissue: an updated review of the clinicopathologic, immunophenotypic, and genetic features. Head Neck Pathol. 2015;9:32-8.

10. Hornick JL, Fletcher CD. Myoepithelial tumors of soft tissue: a clinicopathologic and immunohistochemical study of 101 cases with evaluation of prognostic parameters. Am J Surg Pathol. 2003;27:1183-96.

11. Lee JR, Georgi DE, Wang BY. Malignant myoepithelial tumor of soft tissue: a report of two cases of the lower extremity and a review of the literature. Ann Diagn Pathol. 2007:11:190-8.

12. Antonescu CR, Zhang L, Chang NE, Pawel BR, Travis W, Katabi N, Edelman M, Rosenberg AE, Nielsen GP, Dal Cin P, Fletcher CD. EWSR1POU5F1 fusion in soft tissue myoepithelial tumors. A molecular analysis of sixty-six cases, including soft tissue, bone, and visceral lesions, showing common involvement of the EWSR1 gene. Genes Chromosomes Cancer. 2010:49:1114-24.

13. Le Loarer F, Zhang L, Fletcher CD, Ribeiro A, Singer S, Italiano A, Neuville A, Houlier A, Chibon F, Coindre JM, Antonescu CR. Consistent SMARCB1 homozygous deletions in epithelioid sarcoma and in a subset of myoepithelial carcinomas can be reliably detected by FISH in archival material. Genes Chromosomes Cancer. 2014;53:475-86.

14. Li H, Durbin R. Fast and accurate short read alignment with burrowswheeler transform. Bioinformatics. 2009:25:1754-60. doi:10.1093/ bioinformatics/btp1324. Epub 2009 May 1718

15. DePristo MA, Banks E, Poplin R, Garimella KV, Maguire JR, Hartl C, Philippakis AA, del Angel G, Rivas MA, Hanna M, et al. A framework for variation discovery and genotyping using next-generation DNA sequencing data. Nat Genet. 2011:43:491-8. doi:10.1038/ng.1806. Epub 2011 Apr 1010

16. Jeck WR, Parker J, Carson CC, Shields JM, Sambade MJ, Peters EC, Burd CE, Thomas NE, Chiang DY, Liu W, et al. Targeted next generation sequencing identifies clinically actionable mutations in patients with melanoma. Pigment Cell Melanoma Res. 2014:27:653-63.

17. Cassier PA, Fumagalli E, Rutkowski P, Schoffski P, Van Glabbeke M, DebiecRychter M, Emile JF, Duffaud F, Martin-Broto J, Landi B, et al. Outcome of patients with platelet-derived growth factor receptor alpha-mutated gastrointestinal stromal tumors in the tyrosine kinase inhibitor era. Clin Cancer Res. 2012:18:4458-64.

18. Ding W, Fan XL, Xu X, Huang JZ, Xu SH, Geng Q, Li R, Chen D, Yan GR. Epigenetic silencing of ITGA2 by MiR-373 promotes cell migration in breast cancer. PLoS One. 2015;10:e0135128.

19. Rastrelli M, Passuello N, Cecchin D, Basso U, Tosi AL, Rossi CR. Metastatic malignant soft tissue myoepithelioma: a case report showing complete response after locoregional and systemic therapy. J Surg Case Rep. 2013;16(12). doi:10.1093/jscr/rit109.

20. Bisogno G, Tagarelli A, Schiavetti A, Scarzello G, Ferrari A, Cecchetto G, Alaggio R. Myoepithelial carcinoma treatment in children: a report from the TREP project. Pediatr Blood Cancer. 2014;61:643-6.

21. Rekhi B, Sable M, Jambhekar NA. Histopathological, immunohistochemical and molecular spectrum of myoepithelial tumours of soft tissues. Virchows Arch. 2012:461:687-97.

22. Fletcher CDM, World Health Organization. International Agency for Research on Cancer.: WHO classification of tumours of soft tissue and bone. Lyon: IARC Press; 2013.

23. Indovina P, Pentimalli F, Casini N, Vocca I, Giordano A. RB1 dual role in proliferation and apoptosis: cell fate control and implications for cancer therapy. Oncotarget. 2015;6:17873-90.

24. Etges A, Nunes FD, Ribeiro KC, Araujo VC. Immunohistochemical expression of retinoblastoma pathway proteins in normal salivary glands and in salivary gland tumours. Oral Oncol. 2004;40:326-31.

25. Dowlati A, Lipka MB, McColl K, Dabir S, Behtaj M, Kresak A, Miron A, Yang M, Sharma N, Fu P, Wildey G. Clinical correlation of extensive-stage small-cell lung cancer genomics. Ann Oncol. 2016;27:642-7.

26. Rodriguez ML, Juarez CP, Luna JD. Intravitreal triamcinolone acetonide injection in blind painful eyes. Intraocular steroids as a treatment for blind painful red eyes. Eur J Ophthalmol. 2003;13:292-7.

27. Huang S, Holzel M, Knijnenburg T, Schlicker A, Roepman P, McDermott U, Garnett M, Grernrum W, Sun C, Prahallad A, et al. MED12 controls the response to multiple cancer drugs through regulation of TGF-beta receptor signaling. Cell. 2012;151:937-50.

\section{Submit your next manuscript to BioMed Central and we will help you at every step:}

- We accept pre-submission inquiries

- Our selector tool helps you to find the most relevant journal

- We provide round the clock customer support

- Convenient online submission

- Thorough peer review

- Inclusion in PubMed and all major indexing services

- Maximum visibility for your research

Submit your manuscript at www.biomedcentral.com/submit
Biomed Central 